\title{
Living with Cancer: The Experiences of Chinese Canadian Cancer Survivors
}

\author{
Tsorng-Yeh Lee ${ }^{1}$, F. Beryl Pilkington ${ }^{1} \& \mathrm{Grace}_{\mathrm{Ho}}^{2}$ \\ ${ }^{1}$ School of Nursing, York University, Toronto, Canada \\ ${ }^{2}$ South Riverdale Community Health Centre, 955 Queen St. East, Toronto, Canada \\ Correspondence: Tsorng-Yeh Lee, School of Nursing, York University, \#312 HNES Building, 4700 Keele St. \\ Toronto, ON M3J 1P3, Canada. Tel: 1-416-736-2100 Ext. 20071. E-mail: tsylee@yorku.ca
}

Received: September 15, 2014

doi:10.5539/ijps.v6n4p106

\author{
Accepted: October 21, $2014 \quad$ Online Published: November 10, 2014 \\ URL: http://dx.doi.org/10.5539/ijps.v6n4p106
}

\begin{abstract}
The purpose of this study was to understand the experience, informational and psychosocial needs of Chinese immigrants in Canada who are survivors of breast cancer through individual interviews. A phenomenological approach was used. Eleven Mandarin-speaking Chinese were recruited and interviewed in Toronto, Canada. The interviews were tape-recorded and transcribed verbatim. Data were analysed using Colaizzi's methodology. Seven themes emerged: a) miscommunication between healthcare professionals and participants, b) dissatisfaction with medical professionals, c) lack of cancer related information, d) financial hardship related to cancer medications and living expense, e) emotional changes related to the diagnosis of cancer, f) the dramatic change in lifestyle, and g) the comprehensive support from spouse, family and friends. The result of the study can enhance healthcare providers' understanding of the experience of Chinese Canadian breast cancer survivors and their informational and psychosocial needs. Appropriate intervention strategies in caring cancer patients should be developed.
\end{abstract}

Keywords: Chinese, immigrant, Canada, breast cancer, qualitative research

\section{Introduction}

Breast cancer is the second leading cause of death for women in Canada (Canadian Cancer Statistics [CCS], 2013). In 2013, about 23,800 Canadian women will be diagnosed with breast cancer and 5,000 deaths will occur (CCS, 2013). Cancer patients face profound physical, psychosocial, and financial challenges in their life (Ching, Martinson \& Wong, 2009; Gao, Bennett, Stark, Murray \& Higginson, 2010). Recent cancer studies have shown that many socio-cultural factors impact the outcomes of cancer treatment among minority populations, such as access to health services, lack of knowledge, mistrust, fear, lack of cultural sensitive interventions (Wang, Rosenberg \& Lo, 2008), low health literacy, and patient-provider miscommunication (Clack, Allen, Cooper, \& Head, 2004; Ha \& Longnecker, 2010; Tongue, Epps, \& Forese, 2005). Clack et al. (2004) suggested adopting a more patient-centered service, which can facilitate a good doctor-patient relationship and result in better patient satisfaction as well as doctor satisfaction. Satisfied patients are less likely to complain of malpractice.

The number of Chinese Canadians is growing in Canada; however, there are very few appropriate culture-sensitive or language-friendly resources available for them to obtain in-depth information after being diagnosed with breast cancer (Bell \& Lee, 2010). This lack of information may impede their adjustment by increasing their cancer-related anxiety and degrading their quality of life (Adams, Boulton, \& Watson, 2009).

Kwok and White (2011) conducted a qualitative study to explore the cultural and linguistic isolation of Chinese-Australian breast cancer survivors and found that women in their study suffered a knowledge deficit in breast cancer. Studies have showed that cancer patients' anxiety was relieved and their psychological wellbeing was improved after receiving appropriate cancer information (Booth, Beaver, Kitchener, O'Neill, \& Farrell, 2005; Ziegler, Newell, Stafford, \& Lewin, 2004). Providing information to cancer patients can also empower them and assist them to make necessary treatment decision (Rees, Sheard, \& Echlin, 2003). Lee and colleagues (Lee, Francis, Walker, \& Lee, 2004) found that Chinese breast cancer patients prioritized their information needs. The spread of the cancer, the side effects of chemotherapy and the management of the side effects were what they cared the most. 
An Iranian study (Moradian, Aled avood, \& Tabatabaee, 2012) revealed that patients suffered more from the impact of cancer on their daily life than physical symptoms. Cancer affected their financial situation, ability to work, family life, and expenses of treatment. They rated that expenses of treatment and psychosocial distress were two major factors to impact their daily life. In Canada, the 1984 Canada Health Act provides universal coverage of all insured services to insured persons, such as hospital services, physician services, and surgical-dental services (Health Canada, 2011). However, one of the major disadvantages of the Canadian health care system is that it doesn't cover the cost of medications administered outside a hospital like a cancer medication the patient takes at home (Statistics Canada, 2014). Many patients are very upset to discover they may have to pay out of pocket for their cancer medications that can be extremely expensive.

Hvidt (2013) found that emotional support could assist patients to cope with psychosocial distress related to a diagnosis of cancer. Participants revealed that the sources of their emotional support were the rehabilitation center, the hospital chaplain, fellowship with other cancer survivors, and God/a higher power (Hvidt, 2013). Bell and Lee (2009) emphasized that cancer support groups have been a key mechanism for addressing the psychosocial needs of cancer patients and their families. Not all cancer experiences are negative: A Japanese study (Tsuchiya, Horn, \& Ingham, 2013) showed that cancer survivors often reported positive changes including attitude changes toward life, strengthening trust in family and friends, increased appreciation of life, self-development, future perspectives, education for friends, and efforts towards bodily change. Similarly, a Chinese study found when facing breast cancer, women tried to "make the best of it" (Fu, Xu, Liu, \& Haber, 2008 , p. 155) by actively receiving the cancer treatment, maintaining an optimistic attitude, and moving on. The authors concluded that the Chinese women's experiences with cancer adjustments reflected Chinese historical, social and cultural influences.

It is not known how Chinese women in Canada deal with a diagnosis of breast cancer. Most Chinese Canadians are foreign-born, and thus, may have limited social networks. The language obstacle, cultural adaptations, and the Eastern view of health beliefs and practices may act as barriers to both access to and utilization of, services (Kwok \& White, 2011; Wang et al., 2008). Therefore, the purpose of this study was to understand the experience of Chinese immigrants in Canada who are survivors of breast cancer and their informational and psychosocial needs. The research questions were: a) what are Chinese immigrant women's experiences when they access to Canadian health care services after being diagnosed with breast cancer? and $b$ ) what were their informational and psychosocial needs?

\section{Methods}

A descriptive phenomenological research approach was used in this study. Phenomenology is a research methodology designed to explore and understand people's everyday lived experiences (Streubert \& Carpenter, 2011). It is used to seek the meanings of experiences through engaging in-depth with participants' reality (Lopez $\&$ Willis, 2004), which matches our study purpose.

\subsection{Participants}

Eleven adult female participants were recruited through a well-established Chinese cancer support group in downtown Toronto, Ontario, Canada after obtaining ethical approval from York University. Eligible participants were Mandarin-speaking Chinese women who had a diagnosis of breast cancer. GH, the third author and outreach worker of the support group, identified and contacted eligible participants from a member list. After the women gave verbal agreement to participate, TYL contacted the participants, explained the study purpose and related information, and arranged an interview, which was conducted in Mandarin.

Nine participants were originally from China and two were from Hong Kong. The mean age of the participants was 65.7. The majority of the participants were married and unemployed. Most of them were high school graduates. Three had a university degree. Half of them were religious believers, including Buddhists, Christians, and Taoists.

\subsection{Setting and Data Collection}

The study was conducted at South Riverdale Community Health Centre, Toronto, ON, Canada. Participants were asked to participate in a one-on-one, in-depth interview to explore their cancer experience, and informational and psychosocial needs. Each interview lasted about 60 to 90 minutes. The first author, who has extensive experience in working with breast cancer patients and qualitative research methodology, conducted all of the interviews. The interviews were audio tape-recorded and transcribed verbatim. Each interview was initiated with a broad, open-ended question:" Could you please share with me your experience with a diagnosis of breast cancer?" Participants' thoughts were clarified or expanded if necessary. Nonverbal cues such as gestures and facial 
expressions were observed and noted (Streubert \& Carpenter, 2011). Probing questions, such as "Can you tell me more?" and "What do you mean?" were used. Recruitment of participants continued until no new information emerged from the interview data. An honorarium of $\$ 25$ was given to each participant in appreciation for her participation in this study. The interviews were conducted from March 2012 to April 2013.

\subsection{Data Analysis}

Data were analyzed by the first and third authors of this study: Both of them speak the same language as the participants (Mandarin). Colaizzi's (1978) method was used to analyze the interview data. Each transcript was read and re-read by the two authors to obtain a general sense about the whole content. For each transcript, significant statements were then identified and coded. The coded statements were grouped and transformed into themes, which provided the essential structure of the participants' experiences. A theme was not accepted until agreement by two authors was reached $100 \%$ of the time.

\subsection{Rigor}

Rigor was established using the principles suggested by Lincoln and Guba (1985) which includes credibility, transferability, dependability and confirmability. Member checking was used to establish credibility. Dependability is confirmed in conjunction with credibility (Streubert \& Carpenter, 2011). The first author translated the study results into English and the second author reviewed the findings. Translation into English allows for broader dissemination of the findings, so potential users can read and utilize them, thus promoting transferability (Lincoln \& Guba, 1985). In order to facilitate confirmability, research notes and memos were kept, so that anyone interested can audit the results and follow the research process (Streubert \& Carpenter, 2011). The themes that emerged capturing the cancer survivors' experiences were sent through emails to all the participants for verification and confirmation of the researchers' interpretation of their experiences. All of the participants agreed with the interpretation.

\section{Results}

The following seven themes emerged from the interview data: a) miscommunication between healthcare professionals and participants, b) dissatisfaction with medical professionals, c) lack of cancer related information, d) financial hardship related to cancer medications and living expense, e) emotional changes related to the diagnosis of cancer, f) the dramatic change in lifestyle, and g) the comprehensive support from spouse, family and friends.

Most of the participants expressed that the process from confirming their diagnosis to getting treated was pretty smooth. However, several participants shared their experiences dealing with the healthcare system, including their miscommunication with healthcare professionals and the inexperience of the healthcare professionals.

\section{a) Miscommunication between Healthcare Professionals and Participants}

Miscommunication happened between participants in this study and their healthcare professionals, especially their cancer treatment physicians. Participants misinterpreted what their physician said. They expressed that they thought they clearly understood the physician's instruction; however, the information the physician conveyed and the way the information was perceived were different.

Participant 3, a single mother living with her son, expressed that her experiences with her oncologist was not smooth. Two weeks after her surgery (November), her doctor explained to her that she would need to have chemotherapy and radiotherapy and her chemotherapy would be done in July. Therefore, she thought that her physician would have arranged everything for her and she would just wait for the phone call from the physician. She had waited for five months until she found another tumour on her breast. She then went to see her doctor, who was very surprised that she hadn't done any chemotherapy yet and arranged this for her immediately. She felt that her oncologist was too busy and ignored scheduling her for chemotherapy although she blamed herself for not reconfirming the schedule.

The miscommunication existed not only on treatment planning but also on information seeking. Most of the participants felt that their language limitation meant they either didn't know the question they needed to ask or couldn't use correct terms when they asked their physician. Regarding the communication problem, participant 4, a recurrent breast cancer survivor, stated:

I wrote down every question whenever it emerged, classified and prioritized the questions into different categories. When visiting the physician, if the physician was not busy, I would ask all of the questions I had. If the physician was busy then I just asked the most important questions. 
She said that to obtain a medical report from the physician and carry it everywhere was very important. A similar experience was also stated by participant 1 , who wrote down a memo of her personal disease trajectory for regular visits and emergency use.

\section{b) Dissatisfaction with Medical Professionals}

Participants were referred to their specialists by their family physician. Because of the urgency of their disease, they did not spend much time to check the specialist's background or experience. They could only hope that the specialists who treated them were well qualified and trained in the area in which he or she was practicing. Four out of 11participants in this study expressed their dissatisfaction with the physicians due to their inexperience or lack of certain knowledge.

Participant 3 mentioned:

My specialist took six months to confirm my diagnosis of breast cancer. I felt a tumour in my breast, but the mammogram was negative, so my specialist asked me to wait three months to do a second mammogram. The physician still could not confirm my diagnosis, so two more ultrasounds and a biopsy had been done. The biopsy confirmed my diagnosis.

A similar situation happened to participant 6, who expressed her anger towards her specialist. She did not pay much attention to her biopsy result because her specialist told her that the wound on her breast was "not a big deal". Therefore, she happily went travelling with her husband. It was half a year later she was diagnosed with cancer. She always wondered if things would be different if she could have been treated early.

Participant 2, a 60-year-old single mother, also complained that her physician didn't take good care of her surgery wound by saying:

I felt "hard" on my wound and reported it to my physician, who said that it was a normal healing process and the wound would gradually recover. However, the "hard feeling" was persistent and one year later the wound was infected. I had to visit my physician to drain it out.

Participant 4 was eager to know what treatment options she might have and how big her tumour was; however, her physician was not willing to explain to her. She further explained: "My medical record was just in front of the physician, but the physician did not try to check it at all."

\section{c) Lack of Cancer Related Information}

Most of the participants were not satisfied with the cancer-specific information they had received and were seeking further information to help them deal with the news of the cancer diagnosis. Participant 1 attended lots of workshops to understand the cancer-related information. She stated: "The information my physicians gave to me was not enough and I have to search more information by attending seminars or workshops for the lay population." Several participants appreciated the assistance or information provided by the Chinese cancer support group. Participant 8 , for example, shared that she was very fearful when she was diagnosed with breast cancer. She checked the website and tried to find some useful information; however, a variety of information was there and she didn't know which would fit her situation. Then, she joined the Chinese cancer support group where she met a cancer survivor with the same stage as hers. That person explained to her the whole process of cancer surgery and treatment. She felt calm after the group meeting because she realized what she would go through. Participant 11 also mentioned that she was feeling pretty bad when she was diagnosed with breast cancer, but the more she understood the disease, the more she felt confident in fighting cancer.

\section{d) Financial Hardship related to Cancer Medications and Living Expense}

Although Canadian government health insurance covers most of the cancer treatment expenses, some important health care needs are not; for example, prescription drugs. Participants expressed that they needed to pay many unforeseen and unexpected charges including their cancer medications expenses. All of them were unemployed either before their cancer diagnosis or after the cancer treatment. Therefore, they did not have private drug insurance coverage through a workplace. Most of them depended on their savings or financial support from their husband or children. For example, the only son of participant 3 was studying in university, which aggravated her financial difficulty. She highly appreciated the financial support from the government, such as the Ontario Disability Support Program (ODSP), and Canada Pension Plan Disability Benefits, and not-for-profit organizations like the Breast Cancer Society, etc. However, she also mentioned: "A portion of the money needed to be spent on cancer related products, such as a wig or mastectomy bras and my daily living expense was still a burden to me." She had to get a loan from her ex-husband and so she designated him as the beneficiary of her life insurance because she believed that she would not outlive him. 


\section{e) Emotional Changes Related to the Diagnosis of Cancer}

All of the participants went through a series of emotions when they were confirmed with a diagnosis of cancer. Their emotions included: surprise, sadness, fear, anxiety, acceptance, and calmness. Participant 7 expressed that her emotions and feelings fluctuated enormously from surprise to acceptance when the diagnosis was confirmed. She was very surprised when she was diagnosed with breast cancer because she was quite healthy at that time. She was sad and depressed, but most importantly she was very anxious about how she would be treated and how she could afford the expense related to cancer treatment. The rest of the participants had similar experiences as participant 7. For example, participant 5, whose daughter is a medical doctor, stated:

I was very sad to learn that I had cancer. I was fearful and anxious about my treatment and my survival rate but my daughter told me that cancer is curable. She also explained to me what I would go through. I felt more confident of fighting cancer.

\section{f) The Dramatic Change in Lifestyle}

All of the participants expressed that their lifestyle had changed dramatically because of the diagnosis of cancer, especially their eating habits. They believed certain food causes cancer and tried to avoid these kinds of food as much as they could. Most of them had eaten less meats and fried food since they were diagnosed with breast cancer.

Their attitude toward life had also changed. They said they believed life is short so they would like to enjoy living at present because they were not sure when cancer would take their life. Participant 6 expressed that she used to be stubborn; for example she liked to keep her house clean, neat, and tidy and fought with her husband if he couldn't follow her standard. However, she said she has changed since the diagnosis and now doesn't care much about the cleanness of her house. Her temper also changed from easily angry to more tolerant. Participant 10, a grandmother, mentioned: "Survival is the most important thing in my life right now. My children are all grown up, so my priority is taking good care of myself." Participant 9, a gynaecologist in her home country, stated that when she immigrated to Canada she was eager to find a job in the medical field, however, her English was not good so she found a job in a restaurant and always thought one day she would resume her medical career. She gave up her dream after being diagnosed with breast cancer and thought it would be a blessing if she could happily live every day. She also mentioned that she would like a "good death". She further explained that a good death meant "no pain".

The feeling toward life of two older participants (participants 1 and 11) was different from that of the others. They were more than 80 years old and had a cancer for more than 10 years. They expressed that "fate" was good to them, so they could have such a long life. Both of them were volunteers in a community health care centre. They said they enjoy sharing their life experience to other cancer patients. They often encourage and educate people they encounter to do regular checks in order to be diagnosed and treated early.

\section{g) The Comprehensive Support from Spouse, Family and Friends}

Family members assumed multiple roles in supporting the participants in their fight with cancer. A typical example is participant 6 . Her husband acted as an inspirer, a driver, a cook, a translator, and an information provider. He encouraged her to receive various treatments when she was about to give up. He drove her to every clinic and hospital visit. He has never cooked a meal before his wife's illness but assumed the role of cook. He translated for her when she went to medical checks and he sought information on the internet and translated the information for her. However, not everyone had such a supportive husband. Family members needed to accept the truth that their loved ones needed their assistance. For example, participant 10 mentioned: "In the beginning of my illness, my daughter complained why she needs to assume this burden to financially and emotionally support me, but gradually, she realized that it is the time for her to take care of her mother." Participant 9 stated that her son was her helper who was willing to assist her for transportation and translation. However, her son had to take a leave from work in order to take her to visit her physician.

The cancer support group was another source of support. The participants got useful information from the group and met friends with the same diagnosis. Participant 9 suggested there should be Chinese support groups in the north and west of Toronto. The support group they joined is located in Toronto east. They enjoyed the information exchange and support from group leaders and members, but the lengthy travel sometimes made them tired. 


\section{Discussion}

The results of this study revealed two unique experiences encountered by Chinese immigrants living in Canada which were different from the experience of Canada-born cancer survivors: miscommunication between healthcare professionals and participants and dissatisfaction with medical professionals.

Effective communication between physicians and their patients is very important in the delivery of high-quality health care. A physician who communicates well can facilitate an accurate explanation of the diagnosis, give therapeutic instructions, and help patients follow advice as well as adhere to the prescribed treatment (Ha \& Longnecker, 2010). Miscommunication was rarely mentioned in other cancer studies. The reasons for miscommunication in our study might be due to the language barrier, patients' fear of authority figures, and doctors' burden of work (Ha \& Longnecker, 2010; Wang et al., 2008). A Canadian study with Chinese immigrants done by Wang et al. (2008) mentioned that the family physicians of $96 \%$ of the participants in their study were of Chinese ethnic origin. However, it is not that easy to match a cancer specialist due to the scarcity of Chinese-speaking specialists or geographic location of hospitals. Although hospitals provide some translation services, it is not $24 / 7$ services. Furthermore, some of the translators were not as qualified as expected. In addition, traditionally, Chinese people act as passive recipients and rely on their physicians to make decision for them and they would not challenge the authority of experts (Wang et al., 2008). It is even harder for patients to challenge the specialist's decision given their limited knowledge of either of Canada's official languages, English and French. On the other hand, physicians sometimes discourage patients from expressing their concerns as well as requests for more explanations due to the burden of work (Ha \& Longnecker, 2010). Insufficient explanation may result in poor patient understanding and further impede effective communication between physicians and their patients.

Participants in this study complained about how their physicians managed their care. They hoped that their doctors could skilfully diagnose and treat their illnesses as well as take their chief complaint seriously because early diagnosis is the key point to cancer survival (Kwok \& White, 2011; Tongue et al., 2005). Two possible explanations may explain their dissatisfaction with] physicians. Firstly, some of the physicians may have depended too much on advanced diagnostic techniques and lacked necessary clinical experience to identify patients' problem (Wang et al., 2008). Secondly, the nature of medical education as well as internship and residency training may make physicians tend to lose their focus on holistic patient care and suppress empathy (Clark et al., 2004; Ha \& Longnecker, 2010). However, the complaints may be related to miscommunication and not clinical competency (Clark et al., 2004). Ha and Longnecker (2010) suggested that physicians need to build up better communication and interpersonal skills, so they can detect patients' problems earlier, prevent medical crises and expensive intervention, and provide better support to their patients.

Understanding information related to cancer seems a psychological need for most of the participants in our study, which is consistent with studies done by other researchers (Booth et al, 2005; Ziegler et al., 2004; Lee et al., 2004). Lee and colleagues (2004) reported that cancer patients need information throughout the cancer trajectory. They need to access to health information to help them to understand what outcome they could expect and how to deal with the unexpected challenges. Studies (e.g., Adams et al., 2009) have shown that treatment-related information and prognosis-related information were the most important information that patients need. Not surprisingly, many participants sought to obtain relevant information on the internet. Although the internet offers widespread access to health information, participants preferred to seek face-to-face professional advice through attending workshops and cancer support groups. In the beginning of their journey, participants depended on a support group to provide them treatment information; later on they appreciated the support and understanding from the group members. This is similar to findings from a study in British Columbia (Bell \& Lee, 2010). Due to language limitation, most participants preferred to read Chinese-written information and attend Chinese-speaking workshops and support groups.

The availability of a publicly-funded, universal health care system in Canada alleviates Canadians' health care expenditures; however, there are health care services that are not covered by public health plans, such as cancer medication expenses, which could accumulate quickly and affect cancer survivors' ability to pay for other products they needed. A recent Canadian health report (Statistics Canada, 2014) mentioned that Canadians' out-of-pocket health care spending rose between 1997 and 2009 and the increase was greatest for households in the lowest income quintile. The increase for households in the higher income quintiles ranged from $36 \%$ to $48 \%$; however, the increase in the lowest-income quintile was $63 \%$. The three largest components of out-of-pocket health care expenditures included dental services, prescription medications, and insurance premiums. In general, lower-income families spend more on prescription medications than do higher-income families, who are covered through workplace insurance or can afford private insurance. Many participants in this study were from 
lower-income families; hence, the out-of-packet costs of cancer medications imposed financial hardship on them and their families. In response to this difficulty, many Canadian hospitals have specialized staff to help patients deal with drug reimbursement issues. It is time for the Canadian government to rethink about covering the cost of medications administered outside a hospital, especially for expensive medications, such as cancer medications.

Many studies have been reported the emotional changes when a cancer diagnosis is confirmed (Ching et al., 2009; Fu et al., 2008). Fu et al. (2008) reported that Chinese women with breast cancer tend to try "making the best of it" (p.155); they would typically face the reality, take an active part in the cancer treatment, and sustain an optimistic spirit. Participants in our study also demonstrated similar pattern. Although they had a variety of emotional fluctuation at the beginning of the cancer experience, their level of emotional distress generally decreased over time and by the time of the interview they had subsequently accepted the disease and moved forward to adopt strategies for recovery. They changed their eating habits, temper, and their high standard of everyday living and volunteered to help others by providing advice based on their personal experience. It seemed that participants' level of emotional distress was related to their understanding and appreciation of cancer information, which is consistent with findings of several previous studies that providing information to cancer patients can relieve their distress and improve their psychological wellbeing (Adams et al., 2009; Booth et al., 2005; Lee et al., 2004).

Family members, including husbands and children, provided major support in our study. Bell and Lee (2009) also highlighted the importance of family support. Participants in their study expressed the invaluable moral support from their children and spouses. They also appreciated the sacrifices their children and spouses made in order to provide physical and financial support for them. Group support is another source of support. Participants in our study appreciated the assistance they received from the cancer support group worker. It might be that they wanted to please the worker; however, several participants had attended this group for more than five years. They would not consecutively attend this group if they did not benefit from attending it.

This study has several implications. Given the frequency of miscommunication between patients and health care providers, especially physicians, healthcare providers need to consider improving their communication and interpersonal skills to minimise the risk of communication problems. Healthcare providers need to be aware that misunderstandings can occur at different levels of the communication process and may impact patients' treatment outcomes. A patient-centred or holistic approach to care is recommended. In addition, healthcare providers need to be well-prepared themselves in both understanding of cancer theory and practice principles, so their clinical competency can be improved and mis- or delay diagnosis would not happen. A well-trained and qualified interpreter can bridge linguistic gaps between patients and their healthcare providers, so it is important and necessary to offer an interpreter when possible. Participants in this study seem to be benefiting from attending the Chinese cancer support group. They obtained extra information and support from the group members. However, such groups are rare in Toronto, and so, organizing more Chinese-speaking support groups is suggested.

The study has two limitations. The first limitation is the small sample, which was entirely drawn from a Chinese cancer support group, so the results may not capture the experience of people who do not attend a breast cancer support group. The second limitation is that the average age of the participants was more than 65 years; therefore, the results may not be applicable to a younger population. To better serve the multi-cultural societies in Canada, future studies with other ethnic backgrounds are recommended.

\section{Conclusion}

The results of the study can enhance healthcare providers' understanding of the experiences and informational and psychosocial needs of Chinese Canadian breast cancer survivors. Appropriate intervention strategies in caring for cancer patients from this population should be developed in hospitals and communities to help them to face the physical and psychosocial challenges in the journey of fighting cancer.

\section{References}

Adams, E., Boulton, M., \& Watson, E. (2009). The information needs of partners and family members of cancer patients: A systematic literature review. Patient Education and Counseling, 77, 179-186. http://dx.doi.org/10.1016/j.pec.2009.03.027

Bell, K., \& Lee, J. (2009). Chinese cancer support group study: Key findings. BC Cancer Agency. Retrived from http://www.bccancer.bc.ca/NR/rdonlyres/1DDA0D9C-832C-404A-95B5-7A26719FCBD6/32864/FINALC CSGREPORT9March.pdf 
Bell, K., \& Lee, J. (2010). Is there an "ideal cancer" support group? Key findings from a qualitative study of $\begin{array}{lllll}\text { three grups. Journal of Psychosocial Oncology, 28, 432-449. } & \text {. }\end{array}$ http://dx.doi.org/10.1080/07347332.2010.488140

Booth, K., Beaver, K., Kitchener, H., O’Neill, J., \& Farrell, C. (2005). Women's experiences of information, psychological distress and worry after treatment for gynaecological cancer. Patient Education and Counseling, 56, 225-232. http://dx.doi.org/10.1016/j.pec.2004.02.016

Canadian Cancer Statistics. (2013). National statistics at a glance. Retrieved from $\mathrm{http} / /$ www.cancer.ca/en/cancer-information/cancer-101/canadian-cancer-statistics-publication/?region=bc

Ching, S. S. Y., Martinson, I. M., \& Wong, T. K. S. (2009). Reframing: Psychological adjustment of Chinese women at the beginning of the breast cancer experience. Qualitative Health Research, 19, 339-351. http://dx.doi.org/10.1177/1049732309331867

Clack, G. B., Allen, J., Cooper, D., \& Head, J. O. (2004). Personality differences between doctors and their patients: Implications for the teaching of communication skills. Medical Education, 38(2), 177-186. http://dx.doi.org/10.1111/j.1365-2923.2004.01752.x

Fu, M. R., Xu, B., Liu, Y., \& Haber, J. (2008). 'Making the best of it': Chinese women's experiences of adjusting to breast cancer diagnosis and treatment. Journal of Advanced Nursing, 63, 155-165. http://dx.doi.org/10.1111/j.1365-2648.2008.04647.x

Gao, W., Bennett, M., Stark, D., Murray, S., \& Higginson, I. J. (2010). Psychological distress in cancer from survivorship to end of life care: Prevalence, associated factors and clinical implications. European Journal of Cancer, 46, 2036-2044. http://dx.doi.org/10.1016/j.ejca.2010.03.033

Ha, J. F., \& Longnecker, N. (2010). Doctor-patient communication: A review. The Ochsner Journal, 10, 38-43.

Health Canada. (2011). Canada Health Act-Annual report 2010-2011. Retrived from http://www.hc-sc.gc.ca/hcs-sss/pubs/cha-lcs/2011-cha-lcs-ar-ra/index-eng.php

Hvidt, E. A. (2013). Sources of "relational homes": A qualitative study of cancer survivors' perceptions of emotional support. Mental Health, Religion \& Culture, 16, 617-632. http://dx.doi.org/10.1080/13674676.2012.708652

Kwok, C., \& White, K. (2011). Cultural and linguistic isolation: The breast cancer experience of Chinese-Australian women-A qualitative study. Contemporary Nurse, 39, 85-94. http://dx.doi.org/10.5172/conu.2011.39.1.85

Lee, Y. M., Francis, K., Walker, J., \& Lee, S. M. (2004). What are the information needs of Chinese breast cancer patients receiving chemotherapy? European Oncology Nursing Society, 8, 224-233. http://dx.doi.org/10.1016/j.ejon.2003.12.006

Lopez, K., \& Willis, D. (2004). Descriptive versus interpretive phenomenology: Their contribution to nursing knowledge. Quality Health Research, 14, 726-735. http://dx.doi.org/10.1177/1049732304263638

Moradian, S., Aled avood, S. A., \& Tabatabaee, A. (2012). Iranian cancer patients and their perspectives: a qualitative study. European Journal of Cancer care, 21, 377-383. http://dx.doi.org/10.1111/j.1365-2354.2011.01293.x

Rees, C. E. Sheard, C. E., \& Echlin, K. (2003). The relationship between the information-seeking behaviours and information needs of parents of men with prostate cancer: A pilot study. Patient Education and Counseling, 49, 257-261. http://dx.doi.org/10.1016/S0738-3991(02)00185-4

Statistics Canada. (2014). Trends in out-of-pocket health care expenditures in Canada, by household income, 1997 to 2009. Health Reports, 25, no. 4 (Catalogue number82-003-X).

Streubert, H. J., \& Carpenter, D. R. (2011). Qualitative research in nursing: Advancing the humanistic imperative. Lippincott Williams \& Wilkins, CA: Sage.

Tongue, J. R., Epps, H. R., \& Forese, L. L. (2005). Communication skills for patient centered care: research-based, easily learned techniques for medical interviews that benefit orthopaedic surgeons and their patients. The Journal of Bone and Joint Surgery American, 87, 652-658.

Tsuchiya, M., Horn, S., \& Ingham, R. (2013). Positive changes in Japanese breast cancer survivors: A qualitative study. Psychology, Health \& Medicine, 18, 107-116. http://dx.doi.org/10.1080/13548506.2012.686620 
Wang, L., Rosenberg, M., \& Lo, L. (2008). Ethnicity and utilization of family physicians: A case study of Mainland Chinese immigrants in Toronto, Canada. Social Science \& Medicine, 67, 1410-1422. http://dx.doi.org/10.1016/j.socscimed.2008.06.012

Ziegler, L., Newell, R., Stafford, N., \& Lewin, R. (2004). A literature review of head and neck cancer patients information needs, experiences and views regarding decision making. European Journal of Cancer Care, 13, 119-126. http://dx.doi.org/10.1111/j.1365-2354.2004.00449.x

\section{Copyrights}

Copyright for this article is retained by the author(s), with first publication rights granted to the journal.

This is an open-access article distributed under the terms and conditions of the Creative Commons Attribution license (http://creativecommons.org/licenses/by/3.0/). 